\title{
Simulation of solar radiation during a total eclipse: a challenge for radiative transfer
}

\author{
C. Emde and B. Mayer \\ Institut für Physik der Atmosphäre, Deutsches Zentrum für Luft- und Raumfahrt (DLR), Oberpfaffenhofen, 82234 Wessling, \\ Germany
}

Received: 13 December 2006 - Published in Atmos. Chem. Phys. Discuss.: 15 January 2007

Revised: 27 March 2007 - Accepted: 20 April 2007 - Published: 4 May 2007

\begin{abstract}
A solar eclipse is a rare but spectacular natural phenomenon and furthermore it is a challenge for radiative transfer modelling. Whereas a simple one-dimensional radiative transfer model with reduced solar irradiance at the top of the atmosphere can be used to calculate the brightness during partial eclipses a much more sophisticated model is required to calculate the brightness (i.e. the diffuse radiation) during the total eclipse. The reason is that radiation reaching a detector in the shadow gets there exclusively by horizontal transport of photons in a spherical shell atmosphere, which requires a three-dimensional radiative transfer model. In this study the first fully three-dimensional simulations for a solar eclipse are presented exemplified by the solar eclipse at 29 March 2006. Using a backward Monte Carlo model we calculated the diffuse radiation in the umbra and simulated the changing colours of the sky. Radiance and irradiance are decreased by 3 to 4 orders of magnitude, depending on wavelength. We found that aerosol has a comparatively small impact on the radiation in the umbra. We also estimated the contribution of the solar corona to the radiation under the umbra and found that it is negligible compared to the diffuse solar radiation in the wavelength region from 310 to $500 \mathrm{~nm}$.
\end{abstract}

\section{Introduction}

The astronomical background of a solar eclipse is well understood and the geometry of the problem is known with very high accuracy, e.g. time and location of the Moon's shadow on the Earth as well as its diameter and shape (Espenak and Anderson, 2004). Under cloudless sky conditions one can observe a number of phenomena, for instance the changing colour of the sky, the corona of the sun or the planets and stars which become visible against the darkening sky. Solar

Correspondence to: C. Emde

(claudia.emde@dlr.de) eclipses are primarily of astronomical interest, for instance to take measurements of the corona of the sun (Koutchmy, 1994). On the other hand, a solar eclipse is an excellent means to test radiative transfer models, in particular threedimensional (3-D) radiative transfer codes. There is a specific need to test those against experimental data which is a challenging task: For that purpose, well-characterised threedimensional situations are required; the most prominent ones are inhomogeneous clouds. While the measurement corresponding to the model output (radiation at the ground or in the atmosphere) is a straightforward task, the full characterisation of the input parameters (the cloud properties) with high enough accuracy to actually constrain the model result is close to impossible. A solar eclipse solves this problem to some degree: It is a complex three-dimensional problem, ideally suited to test the accuracy of the code: Radiation reaching a detector under the shadow gets there exclusively by horizontal (three-dimensional) transport of photons in a spherical shell atmosphere. The model input (distribution of the incoming solar radiation at top-of-atmosphere) is known with high accuracy. As it turns out, the situation for a solar eclipse is reversed compared to the broken cloud case: While the input conditions are easily available, measuring the output (radiation under the shadow) is actually a challenging experimental problem which requires careful planning.

Several radiation measurements during total eclipses have been carried out, mainly in the 1960s and 1970s (Sharp et al., 1971; Silverman and Mullen, 1975). Recent spectral measurements were evaluated only for partial eclipses or in the pre-umbra (Fabian et al., 2001; Aplin and Harrison, 2003). Shaw (1978) developed a greatly simplified radiative transfer model wherein sunlight diffuses into the umbra only by first- and second-order scattering processes. According to Shaw this model can calculate the diffuse radiance in the umbra within an uncertainty of a factor of two. Koepke et al. (2001) used a one-dimensional (1-D) radiative transfer model for simulations in the pre-umbra. Both models account for

Published by Copernicus GmbH on behalf of the European Geosciences Union. 

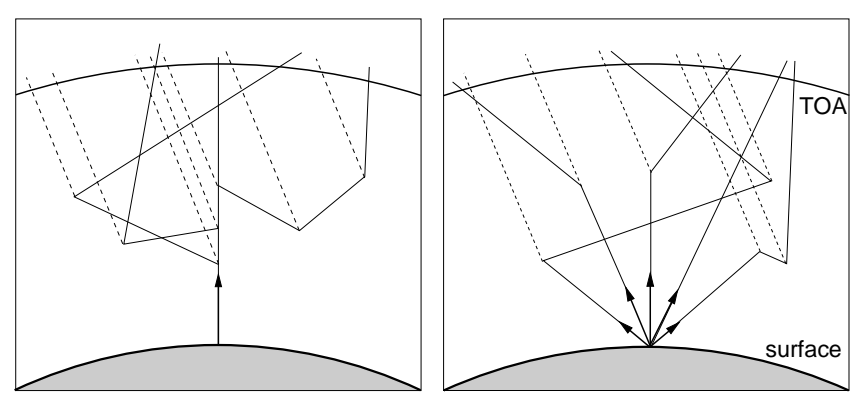

Fig. 1. Typical photon paths in backward tracing. The left panel illustrates the calculation of solar zenith radiance and the right panel the calculation of diffuse solar irradiance. Solid lines are actual photon trajectories and dashed lines are the contributions to the radiance/irradiance according to the local estimate technique, see text.

solar limb darkening. As mentioned above, a 3-D multiple scattering model is required to model the solar radiation inside the umbral shadow accurately. Monte Carlo methods can be used for such applications (e.g., Marshak and Davis, 2005). For this particular problem, forward Monte Carlo calculations are very time consuming because only a small fraction of the photons started outside the shadow reach a sensor placed in the centre of the umbral shadow, which results in large uncertainties or very long computation times. Hence, backward Monte Carlo calculations are appropriate where all photons are started at the sensor position and followed backwards towards the top of the atmosphere. As our sensitivity studies prove, a spherical model atmosphere is required because light entering the atmosphere more than $1000 \mathrm{~km}$ away may impact the radiance or irradiance in the centre of the umbral shadow.

Here we describe the radiative transfer code, in particular the specialities required for the eclipse simulation: backward Monte Carlo calculations and spherical geometry. We then present quantitative spectral radiance and irradiance calculations for an example location: the Greek island Kastelorizo $\left(36.150^{\circ} \mathrm{N}, 29.596^{\circ} \mathrm{E}\right)$ which was close to the centre of the umbra, see also Blumthaler et al. (2006). The results and methodology presented here give an overview of the radiance and irradiance levels to be expected during a total solar eclipse and may serve as a benchmark for planning radiation observations during future solar eclipses, e.g. to focus on the wavelength regions where the radiation is well above the detection limit of the instrument, or to optimise integration time.

The following section describes the methodology used to calculate the diffuse radiation in the umbra. In Sect. 3 we show the results of our calculations and in Sect. 4 we summarise our conclusions.

\section{Methodology}

In this section we first describe the radiative transfer model used for this study. With the backward Monte Carlo code we calculate the contribution of each location at top-ofatmosphere to the radiance/irradiance at the centre of the umbra. Then, the incoming (extraterrestrial) irradiance distribution at top-of-atmosphere is derived under eclipse conditions. The product of both (contribution function and extraterrestrial irradiance distribution) gives the radiance/irradiance below the umbra.

\subsection{Monte Carlo model}

The radiative transfer model MYSTIC (Monte Carlo code for the phYSically correct Tracing of photons In Cloudy atmospheres) (Mayer, 1999, 2000) is used for this study. MYSTIC is operated as one of several radiative transfer solvers of the libRadtran radiative transfer package by Mayer and Kylling (2005). Originally MYSTIC has been developed as a forward tracing method for the calculation of irradiances and radiances in 3-D plane-parallel atmospheres. For this study, the model has been extended to allow backward Monte Carlo calculations in spherical geometry. These extensions were required for accurate simulations in the centre of the umbra. In the following we describe only those model properties which are afterwards required to interpret the results of this paper. For general questions about the Monte Carlo technique and in particular about libRadtran and MYSTIC the reader is referred to the literature (Mayer, 1999, 2000; Mayer and Kylling, 2005; Marshak and Davis, 2005; Cahalan et al., 2005).

\subsubsection{Backward Monte Carlo method}

Figure 1 illustrates some typical photon paths through the atmosphere. In a backward simulation, photons are started at the location of the sensor and traced through the atmosphere where they may be scattered or absorbed, until they leave to space at top-of-atmosphere (TOA) or are absorbed at the surface. The reciprocity principle allows to treat scattering and absorption processes in the same way in forward and backward calculations (Marshak and Davis, 2005; Chandrasekhar, 1950). At each scattering, a new direction is randomly sampled from the scattering phase function at each particular location. Absorption is considered by reducing the photon weight according to Lambert-Beer's law along the photon trajectory. The solid lines in Fig. 1 show typical photon paths. For radiance calculations (left) all photons are started into the viewing direction which is the zenith in this example. One can easily imagine that only few photons leave TOA into the direction of the sun. Rather than sampling only these few photons in a very narrow solid angle cone, a much more efficient approach is used: In the "local estimate" technique (Marshak and Davis, 2005) we calculate 


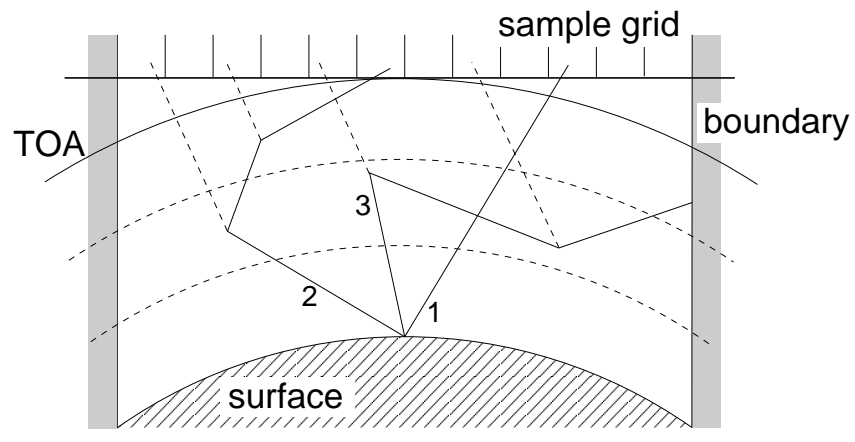

Fig. 2. Backward Monte Carlo sampling. The local estimate method is applied in a spherical atmosphere with absorbing boundary conditions. Contributions are sampled in the TOA reference plane.

the probability that the photon is actually scattered into the direction of the sun at each scattering point. Extinction along the virtual path to the sun is again considered by reducing the photon weight according to Lambert-Beer's law - this time for extinction rather than absorption. The sum of all individual contributions (indicated by the dashed lines in Fig. 1) converges much faster to the desired radiance value than the direct sampling. For diffuse irradiance calculations photons are started in a random direction, with a probability proportional to the cosine of the polar angle (right panel of Fig. 1). Otherwise, the procedure is identical to the radiance calculation.

The photon counting is illustrated in Fig. 2. Photons are binned into rectangular bins at a reference plane at top-ofatmosphere. Sampling in a reference plane allows straightforward weighting with the extraterrestrial irradiance afterwards (which would usually be constant with location but varies of course in the case of a solar eclipse). To get the radiance or irradiance at the sensor position all sampled photons at TOA are multiplied with the extraterrestrial irradiance, integrated (summed) over area, and normalised to the total number of traced photons. While it would in principle be possible to directly add all contributions to get the radiance/irradiance, we will show later that storing the full distribution has a large advantage for solar eclipse simulations. What the distribution actually tells us is where each photon reaching the detector came from, or, the contribution of each location in the TOA reference plane to the radiance/irradiance at the detector. For this reason we will call the function the "contribution function" in the following text.

\subsubsection{Photon tracing in a spherical shell atmosphere}

To introduce spherical geometry into MYSTIC, two issues had to be considered. First, for tracing of photons we need to repeatedly calculate the step width $s$ to the next spherical shell. Figure 3 shows the Cartesian coordinate system used

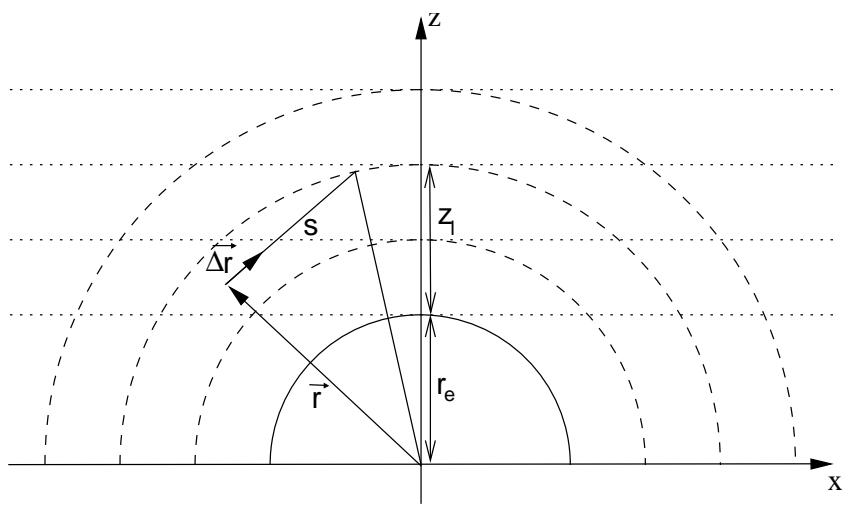

Fig. 3. Coordinate system. The paths of photons travelling in the a spherical shell atmosphere are calculated in Cartesian coordinates.

by MYSTIC even in spherical geometry. $s$ is calculated by solving

$$
\left(\boldsymbol{r}_{\boldsymbol{p}}+s \boldsymbol{\Delta} \boldsymbol{r}\right)^{2}=\left(z_{l}+r_{e}\right)^{2}
$$

where $\boldsymbol{r}_{\boldsymbol{p}}$ is the vector pointing from the Earth's centre to the position of the photon, $\boldsymbol{\Delta} \boldsymbol{r}$ is the normalised direction vector of the photon, $z_{l}$ is the altitude coordinate, counted from the boundary to the next layer which the photon path intersects, and $r_{e}$ is the Earth's radius. The solutions found for up- or downward travelling photons are

$$
s_{\mathrm{down} / \mathrm{up}}=\frac{\boldsymbol{r}_{\boldsymbol{p}}{ }^{2}-\left(z_{l}+r_{e}\right)^{2}}{-\boldsymbol{r} \cdot \boldsymbol{\Delta} \boldsymbol{r} \pm \sqrt{(\boldsymbol{r} \cdot \boldsymbol{\Delta} \boldsymbol{r})^{2}-\boldsymbol{r}_{\boldsymbol{p}}^{2}+\left(z_{l}+r_{e}\right)^{2}}} .
$$

If the photon is travelling downwards and touches the layer below tangentially the denominator of this equation becomes zero. From simple geometrical considerations we find for this special case

$s=-2 \boldsymbol{r} \cdot \boldsymbol{\Delta} \boldsymbol{r}$.

Please note that Eq. (2) is the numerically stable solution of the quadratic equation. The usual textbook solution is illposed and often fails due to the limited accuracy of numerical computations.

The second issue to consider are the boundary conditions. While in plane-parallel geometry periodic boundary conditions are commonly used and are very convenient (as they guarantee energy conservation), this assumption is not reasonable in a spherical-shell atmosphere. Here the only reasonable alternative are absorbing boundary conditions where a photon which hits the boundary is destroyed. This assumption is of course not physically correct; therefore one has to make sure that the domain is sufficiently large, so that the lost photons are negligible. An alternative would be to include the whole earth into the domain. Usually this is not an issue because the sampling domain can be just one large bin, except for the solar eclipse simulation where we want to store the spatially resolved contribution function. 

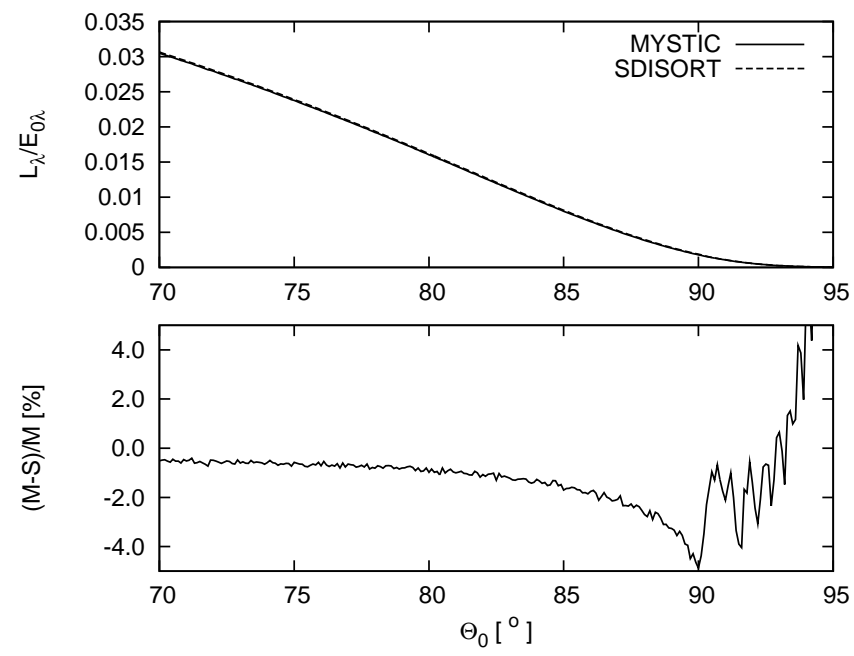

Fig. 4. Comparison between spherical MYSTIC and SDISORT. Zenith radiance at $342 \mathrm{~nm}$ as a function of solar zenith angle. (Top) Radiance normalised by the extraterrestrial irradiance; (bottom) relative deviation between MYSTIC and SDISORT in percent.

\subsubsection{Model validation}

The newly developed backward Monte Carlo model was validated by comparison with the well-tested MYSTIC forward model in three-dimensional geometry. The models agreed perfectly within the Monte Carlo noise of much less than $1 \%$ for all cases tested. The forward MYSTIC model was validated extensively within the Intercomparison of 3-D Radiation Codes (I3RC) (Cahalan et al., 2005). The spherical Monte Carlo model was compared to the pseudo-spherical model SDISORT by Dahlback and Stamnes (1991).

A typical result is shown in Fig. 4. Here, zenith radiances are calculated at $\lambda=342 \mathrm{~nm}$. The surface albedo was 0.06 , typical for ocean. No aerosol was included in the calculation. Up to a solar zenith angle of $80^{\circ}$ the difference between the models is below $1 \%$ and up to $90^{\circ}$ it is within $5 \%$. Such differences may be expected because SDISORT is a pseudospherical code which does not work accurately for very low sun. For solar zenith angles above $90^{\circ}$, e.g. for twilight calculations, the uncertainty of SDISORT increases.

\subsubsection{Example for the contribution function at TOA}

In the following we show the TOA contribution function sampled by the spherical backward Monte Carlo code outlined in the previous sections. As described above, this function describes where the photons which arrive at the detector came from. As an example, we show the contribution function at TOA for a wavelength of $340 \mathrm{~nm}$. For this simulation we used a domain size of $1000 \times 1000 \mathrm{~km}^{2}$. The mid-latitude summer atmosphere by Anderson et al. (1986) was used for the pressure, temperature, and trace gas profiles. The atmosphere was cloudless and aerosol-free. A solar zenith angle
Table 1. Parameters describing the total eclipse at 29 March 2006; from Espenak and Anderson (2004).

\begin{tabular}{cccc}
\hline $\mathrm{t}[\mathrm{s}]$ & $\mathrm{UTC}$ & latitude & longitude \\
\hline-300 & $10: 50$ & $34^{\circ} 40.8^{\prime} \mathrm{N}$ & $28^{\circ} 33.7^{\prime} \mathrm{E}$ \\
0 & $10: 55$ & $36^{\circ} 13.3^{\prime} \mathrm{N}$ & $30^{\circ} 33.5^{\prime} \mathrm{E}$ \\
300 & $11: 00$ & $36^{\circ} 46.7^{\prime} \mathrm{N}$ & $32^{\circ} 34.6^{\prime} \mathrm{E}$ \\
& & & \\
$\mathrm{t}[\mathrm{s}]$ & $\rho$ & $\theta_{0}\left[^{\circ}\right]$ & $\phi_{0}\left[^{\circ}\right]$ \\
\hline-300 & 1.0499 & 32.6 & 18.5 \\
0 & 1.0494 & 35.0 & 23.3 \\
300 & 1.0489 & 37.6 & 27.8 \\
& & & \\
$\mathrm{t}[\mathrm{s}]$ & & Major axis $[\mathrm{km}]$ & Minor axis $[\mathrm{km}]$ \\
\hline-300 & & 196.2 & 165.5 \\
0 & & 200.0 & 164.1 \\
300 & & 204.7 & 162.5 \\
\hline
\end{tabular}

$\theta_{0}$ of $35.0^{\circ}$ was assumed and the solar azimuth angle $\phi_{0}$ was $23.3^{\circ}$ (South-West, or lower-left in the image), corresponding to the conditions during the eclipse of 29 March 2006, 10:55 UTC, at Kastelorizo (see Table 1).

Figure 5 shows the contribution function sampled at TOA. The right panel shows a zenith radiance calculation. The most striking feature $-\mathrm{a}$ bright line along the direction of the solar azimuth - is easily understood: A large part of the radiance in a cloudless atmosphere at $340 \mathrm{~nm}$ stems from single-scattered photons. At $340 \mathrm{~nm}$ the vertically integrated optical thickness $\tau$ of the assumed Rayleigh atmosphere is 0.713. According to Lambert-Beer's law a fraction of $1-\exp \left(-\tau / \cos \theta_{0}\right)=0.58$ of the incoming photons is scattered along their direct path to the surface and the chance for being scattered a second time is comparatively small.

Figure 6 illustrates that photons arriving at the detector after only one scattering event enter the atmosphere along a straight line between points $P 1$ and $P 2 . P 1$ is the spot where a photon directly arrives at the detector without scattering (which for zenith radiance is of course a limiting case that does not actually occur). $P 1$ is close to the bright spot in Fig. 5 which indicates the maximum contribution - related to a scattering close to the surface. $P 2$ is the other extreme where a photon is scattered at top-of-atmosphere to reach the detector. This is a highly unlikely event (due to the exponentially decreasing Rayleigh scattering coefficient with height) for which reason the visible line in the contribution function thins out and probably never reaches $P 2$. The "halo" around the single-scattering line is caused by multiple scattering. As expected, the contribution function drops quickly as we move away from the single-scattering line. Please note that a logarithmic grey-scale was chosen for this plot because otherwise the contribution of the multiply scattered photons would be barely visible. The left panel shows the contribution function 

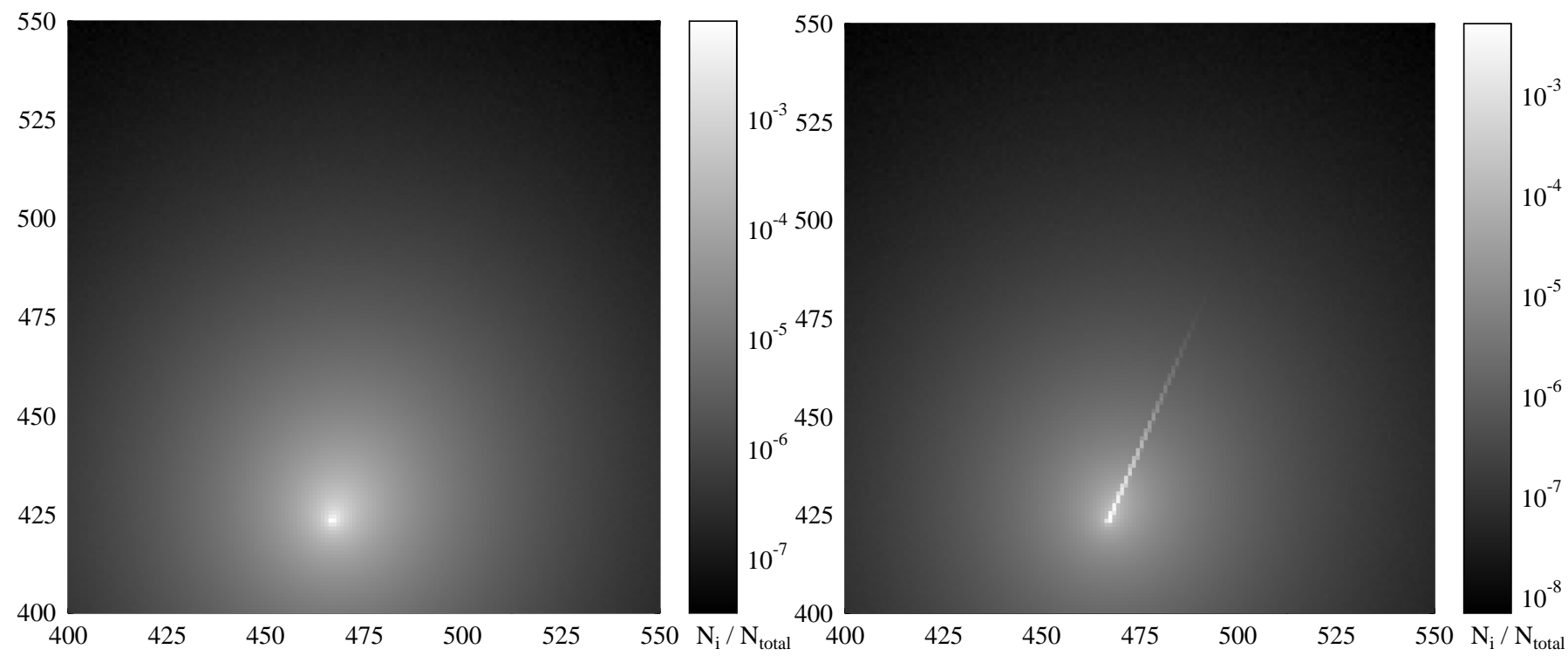

Fig. 5. Distribution of photons at TOA (Contribution function). The left panel shows the calculation of irradiance and the right panel the calculation of zenith radiance. The number of photons in the sample bins $\mathrm{N}_{i}$ divided by the total number of photons is shown. The $\mathrm{x}$ - and $\mathrm{y}$-axes correspond to the coordinates of the sample domain, which is $1000 \times 1000 \mathrm{~km}^{2}$ in this example. The observer is placed in the centre of the domain at $(500 \mathrm{~km}, 500 \mathrm{~km})$. Note that only a part of the domain is shown.

for the diffuse irradiance calculation. The scattered photons produce an approximately radially symmetric pattern about $P 1$ because the single-scattered photons in the irradiance case may enter basically anywhere in the domain (since photons from arbitrary directions contribute to the irradiance in contrast to the radiance).

\subsection{Incoming solar irradiance during the eclipse}

The second essential information in addition to the contribution function required to calculate radiance/irradiance under solar eclipse conditions is the distribution of the extraterrestrial solar irradiance in the reference plane at top-ofatmosphere. Obviously, the extraterrestrial irradiance is zero in the umbra and increases with distance from the umbra.

The incoming solar irradiance depends mainly on the fraction of the sun covered by the moon. The irradiance decrease depends also on wavelength because of the solar limb darkening. Koepke et al. (2001) derived a formula describing the incoming irradiance of the partly covered sun disk $I_{C \lambda}$ as a function of the distance $X$ between the centres of apparent sun and moon disks. We closely follow the formulation by Koepke et al. (2001) but for convenience use the distance normalised to the apparent sun radius $R_{M}$. At the "first contact" the distance between the disk centres is equal to the sum of apparent moon radius $R_{M}$ and apparent sun radius $R_{S}$. Per definition $X$ is negative at that point, therefore $X=-\left(R_{M}+R_{S}\right) / R_{S}$. When the centres of the disks coincide $X=0$. At the "fourth contact" (when the disk of the

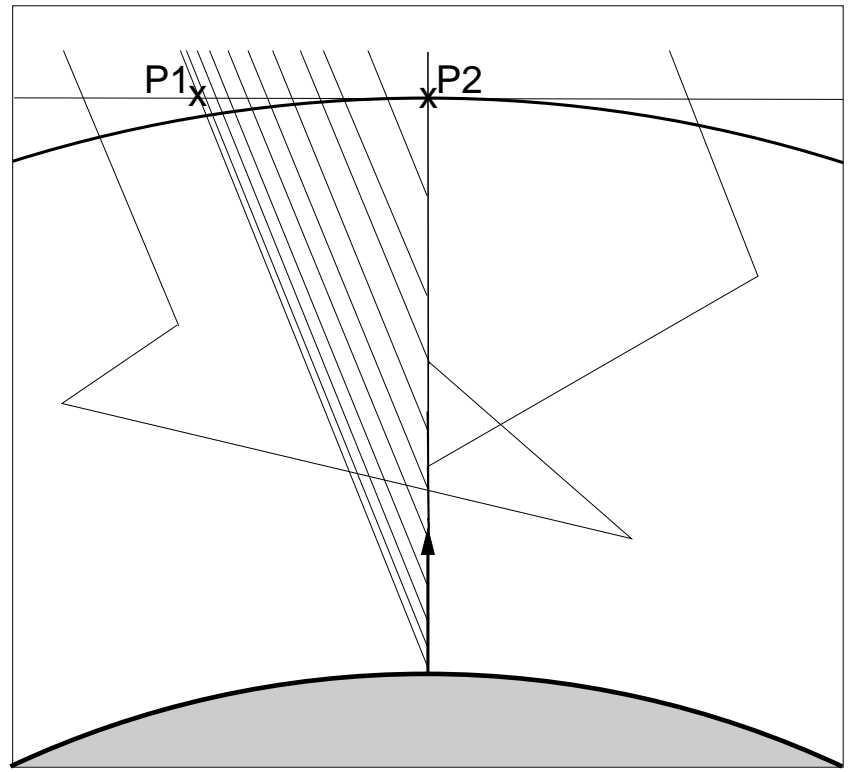

Fig. 6. Photon paths for zenith radiance calculations. All single scattered photon enter the atmosphere between points $P 1$ and $P 2$.

moon leaves the sun disk completely), $X=\left(R_{M}+R_{S}\right) / R_{S}$. For the total solar eclipse from 29 March 2006, the ratio of apparent radii is $\rho \equiv R_{M} / R_{S}=1.0494$ according to Espenak and Anderson (2004) (see also Table 1). Totality occurs for $-\left(R_{M}-R_{S}\right) / R_{S} \leq X \leq\left(R_{M}-R_{S}\right) / R_{S}$, i.e. for $1-\rho \leq X \leq \rho-1$. 


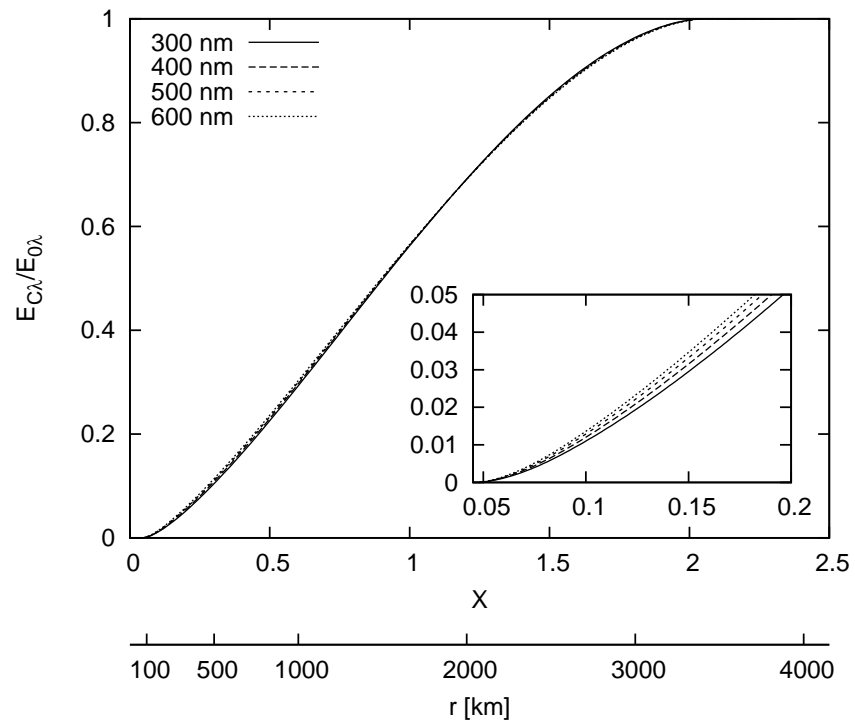

Fig. 7. Incoming solar irradiance during an eclipse. The irradiance is plotted as a function of distance between the centres of the apparent sun and moon disks; the lower axis gives the corresponding distance from the centre of the umbra in $\mathrm{km}$. The irradiance is normalised to its non-eclipse value.

Figure 7 shows the solar irradiance for different wavelengths as a function of $X$ for the solar eclipse from 29 March 2006, calculated according to Koepke et al. (2001). As expected it is zero for $X<0.0494(=\rho-1)$. Only the part for positive $X$ is shown because the irradiance is symmetric about $X=0$. The small figure shows that the wavelength dependence due to solar limb darkening is important for small $X$. For radiation calculations inside the umbral shadow this might be important because these are the photons entering the atmosphere closest to the point of interest, below the umbra.

As a final step we need to project the irradiance distribution from Fig. 7 onto the model reference plane at TOA. For that purpose, we first need to convert from relative distance $X$ to absolute distance in the reference plane. According to Espenak and Anderson (2004)(see Table 1) the width of the minor axis of the umbral shadow at 10:55 UTC is $164.1 \mathrm{~km}$ corresponding to $X=2 \cdot(\rho-1)$. With $\rho=1.0494$ we find that $X=1$ corresponds to $1661 \mathrm{~km}$ which allows us to linearly translate between $X$ and distances in the reference plane (please note that the data of Espenak and Anderson (2004) refer to the surface of the Earth; our reference plane is TOA instead but the $120 \mathrm{~km}$ difference may be safely neglected compared to the distance between Earth and Moon for this application). We used this relationship to provide the second axis in Fig. 7 which shows the distance in $\mathrm{km}$ to give an idea over which distances from the centre of the umbra the incoming solar irradiance is actually disturbed by the Moon's shadow. Finally, we project the thus-derived distribution onto the TOA reference plane and obtain the incoming solar irradiance as a function of the coordinates $x$ and $y$ describing any point the reference plane. In the following we call

$w(x, y)=\frac{E_{C \lambda}(x, y)}{E_{0 \lambda}}$

the solar eclipse weighting function.

Multiplication of the contribution function (see Fig. 5) with $w(x, y)$ gives the actual contribution of each location in the TOA reference plane to the radiance/irradiance at the centre of the umbra. The left panel of Fig. 8 shows the result for the irradiance, the right panel the respective radiance data. First, we note that the absolute values are several orders of magnitude smaller than in Fig. 5. This is due to the fact that the main contributions to the radiance/irradiance at the ground (single and other low orders of scattering) are suppressed by the Moon's shadow. Second, in contrast to Fig. 5 the weighted distributions are plotted on a linear scale which shows that the decrease towards the border of the domain is much slower and that even photons entering the atmosphere more than $300 \mathrm{~km}$ away from the sensor might contribute significantly to the result. We find that the rapid decrease in the contribution function away from the umbra is partly compensated by the increase of the incoming solar irradiance. And third, the contribution to radiance and irradiance look rather similar in contrast to the eclipse-free conditions in Fig. 5. To obtain absolute values of the radiance and irradiance we simply integrate the data from Fig. 8 over the domain and multiply with the extraterrestrial irradiance $E_{0 \lambda}$.

\subsection{Domain size}

The choice of the domain size is directly related to the question how far the photons travel through the atmosphere. For normal (non-eclipse) conditions and high sun angles small domain sizes are sufficient because most of the measured photons have entered the atmosphere close to the point where the direct beam to the receiver hits TOA. They reach the sensor directly or after only a few scattering events in the troposphere as seen in Fig. 5. However, in our application the solar eclipse weighting function (4) masks those photons and gives preference to photons which enter the domain far away from the receiver. The incoming solar irradiance increases rapidly with the distance $r$ from the centre of the umbra. In addition the annular area between $r$ and $r+\Delta r$ increases linearly with distance. In order to find an appropriate domain size, calculations for sizes up to $7000 \times 7000 \mathrm{~km}^{2}$ were performed for $\lambda=342 \mathrm{~nm}$. The results are shown in Fig. 9. The error bars are 2 standard deviations of the result to quantify the Monte Carlo noise. For domains smaller than $1000 \times 1000 \mathrm{~km}^{2}$ one obviously gets wrong results for radiances and irradiances because too many photons are absorbed at the boundary of the domain. We decided to use a domain size of $3000 \times 3000$ $\mathrm{km}^{2}$ for the solar eclipse simulations to be on the safe side. In consequence a spherical radiative transfer model is required 


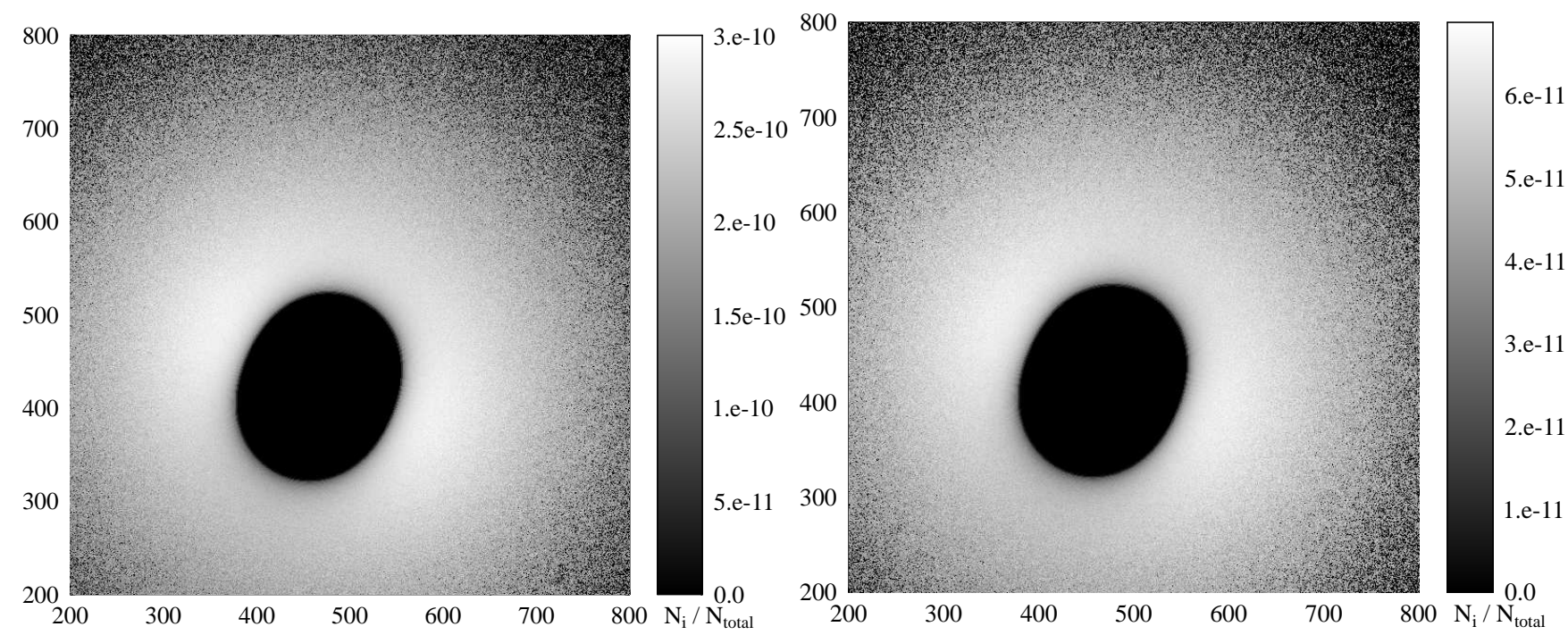

Fig. 8. Weighted contribution function at TOA. The left panel shows the calculation of irradiance and the right panel the calculation of zenith radiance in the centre of the umbral shadow. The $\mathrm{x}$ - and $\mathrm{y}$-axes correspond to the coordinates of the sample domain, which is $1000 \times 1000 \mathrm{~km}{ }^{2}$ in this example. The observer is placed in the centre of the domain at $(500 \mathrm{~km}, 500 \mathrm{~km})$. Note that only a part of the domain is shown.

for such calculations because for such large domains the curvature of the Earth has to be taken into account.

\section{Results}

\subsection{General setup}

This section describes the setup which is common for all calculations shown below. As in the example shown in Sect. 2.1.4 atmospheric pressure, temperature, and trace gas profiles were taken from the mid-latitude summer atmosphere by Anderson et al. (1986). The following parameters were chosen according to the conditions of the total solar eclipse on 29 March 2006 at the Greek island Kastelorizo at approximately 10:55 UTC (see Table 1): The surface albedo was set to 0.06 which is a typical value for an ocean surface. The ozone column was rescaled to $302 \mathrm{DU}$ corresponding to the OMI (Ozone Monitoring Instrument, http: //toms.gsfc.nasa.gov/ozone/ozone_v8.html) measurement at the specific day and location. The sun position was $\theta_{0}=35.0^{\circ}$ (solar zenith angle) and $\phi_{0}=23.3^{\circ}$ (solar azimuth angle). As mentioned in the last section the domain size for the calculation was $3000 \times 3000 \mathrm{~km}^{2}$ with a sample resolution of $3 \times 3 \mathrm{~km}^{2}$. To calculate the short-wave spectrum 20 wavelengths in the range from 300 to $500 \mathrm{~nm}$ were calculated. The results were then interpolated and multiplied with the extraterrestrial solar spectrum which is the standard procedure in libRadtran (Mayer and Kylling, 2005). $10^{7}$ photons were traced for each wavelength.
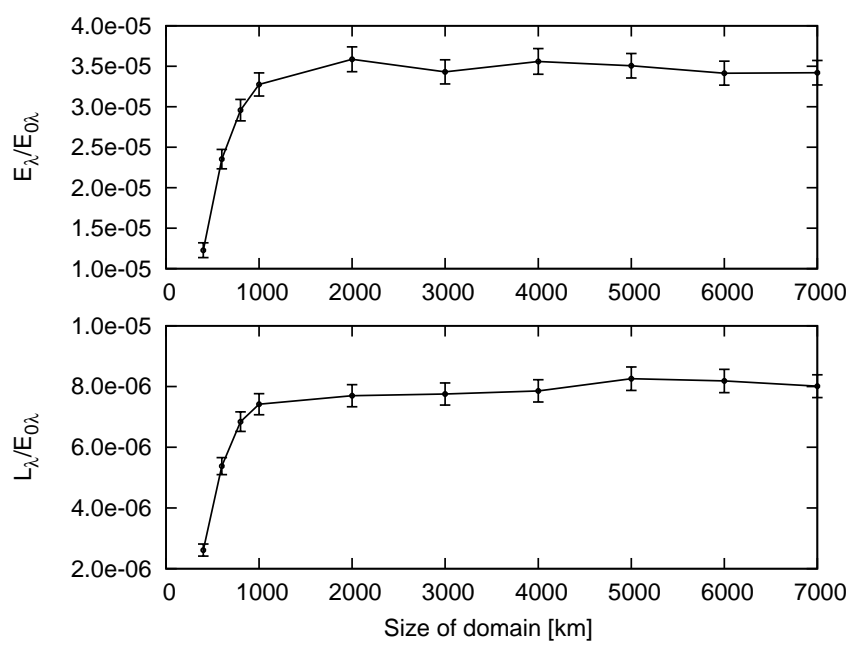

Fig. 9. Impact of domain size at wavelength $\lambda=342 \mathrm{~nm}$.

\subsection{Radiance and irradiance spectra}

The irradiance spectrum at the ground in the centre of the umbral shadow is shown in the left panel of Fig. 10 and Fig. 11 shows the ratios between irradiance and radiance values under non-eclipse conditions and the respective values in the centre of the umbra.

Below $330 \mathrm{~nm}$ the irradiance is strongly reduced due to ozone absorption, enhanced by the long path through the atmosphere, comparable to the pathlength enhancement due to multiple scattering in optically thick clouds (Mayer et al., 1998). The solar radiance spectrum for a zenith viewing 

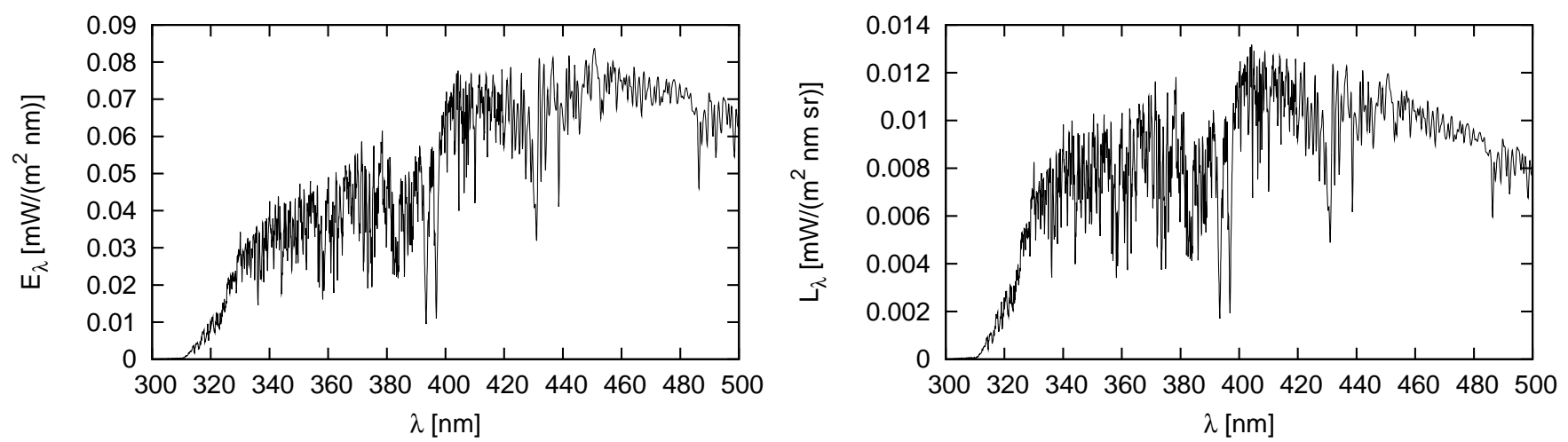

Fig. 10. Spectral irradiance (left) and zenith radiance (right) during the eclipse.
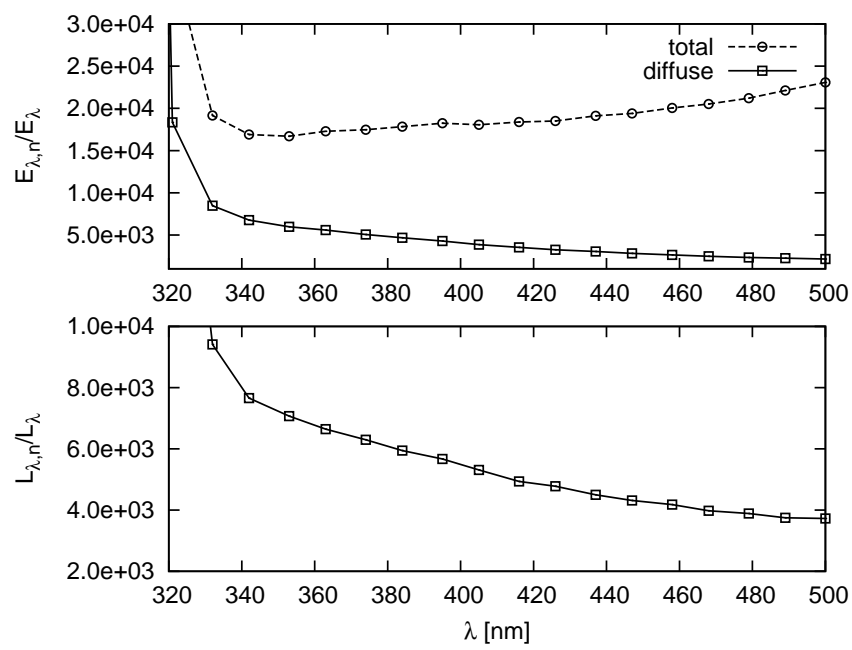

Fig. 11. Enhancement of irradiance (top) and zenith radiance (bottom) during the eclipse. Square markers show the ratios between non-eclipse $\left(E_{\lambda, n}, L_{\lambda, n}\right)$ and eclipse $\left(E_{\lambda}, L_{\lambda}\right)$ calculations for diffuse irradiance and radiance and circles show the same ratio for total irradiance.

instrument at the ground in the centre of the umbral shadow is shown in the right panel of Fig. 10. A decrease at larger wavelengths is observed for zenith radiances. Using the Monte Carlo code we may look at individual photon paths. We found that photons reaching the centre of the umbra mostly get there after two scattering processes: After entering the atmosphere outside the umbra, they are first scattered at a high altitude. Then they travel a long distance horizontally through the optically thin higher atmosphere into the umbra. In the centre of the umbra they undergo the second scattering process, downward towards the sensor. The results show that these second-order scattering processes are less wavelength-dependent than the single-scattering processes which dominate the radiance under non-eclipse conditions.

Figure 11 shows that the total irradiance is decreased by a factor of about 20000 at $330 \mathrm{~nm}$ and by a factor of 23000 at $500 \mathrm{~nm}$. For total irradiance the ratio has a minimum at about $350 \mathrm{~nm}$. This has to do with the direct radiance which increases with wavelength under non-eclipse conditions and which is removed during the total eclipse. For diffuse irradiance the ratio decreases continuously from about 8500 at $330 \mathrm{~nm}$ to 2100 at $500 \mathrm{~nm}$ and for radiance from 9500 at $330 \mathrm{~nm}$ to 3700 at $500 \mathrm{~nm}$. The order of magnitude of those calculations is comparable to the results obtained by Shaw (1975) and to the measurements described in Silverman and Mullen (1975); Sharp et al. (1971).

\subsubsection{Effect of aerosol}

To test the influence of aerosol in the atmosphere a standard aerosol model (Shettle, 1989) was assumed. A rural type aerosol is included in the boundary layer and background aerosol above $2 \mathrm{~km}$. Spring-summer conditions were selected and the horizontal visibility was set to $50 \mathrm{~km}$ which yields a vertically integrated optical thickness of 0.263 at $340 \mathrm{~nm}$ and 0.162 at $550 \mathrm{~nm}$. Results are shown in Fig. 12. In non-eclipse conditions the impact of aerosol on diffuse radiation can be considerable. As seen in the figure the zenith radiance is enhanced by a factor of three at $500 \mathrm{~nm}$ when aerosol is included. Under the umbra, however, the irradiance and radiance are much less affected by aerosol: While the diffuse irradiance is reduced by up to $18 \%$ at $500 \mathrm{~nm}$, the effect of aerosol on the radiance is even smaller, only up to $4 \%$. The reason again lies in the very different pathways of radiation under eclipse and non-eclipse conditions: Under non-eclipse conditions the aerosol is the main source for the diffuse irradiance and radiance in addition to Rayleigh scattering. Non-absorbing aerosol may therefore increase both quantities considerable, in particular at larger wavelengths where the Rayleigh scattering coefficient is small. Under the umbra, however, as we explained above, the main source of radiation at the detector is Rayleigh scattering in the stratosphere, well above the aerosol layer, and the role of aerosol is therefore reduced to attenuating the diffuse radiation on its way from the stratosphere to the detector. This suggests, 

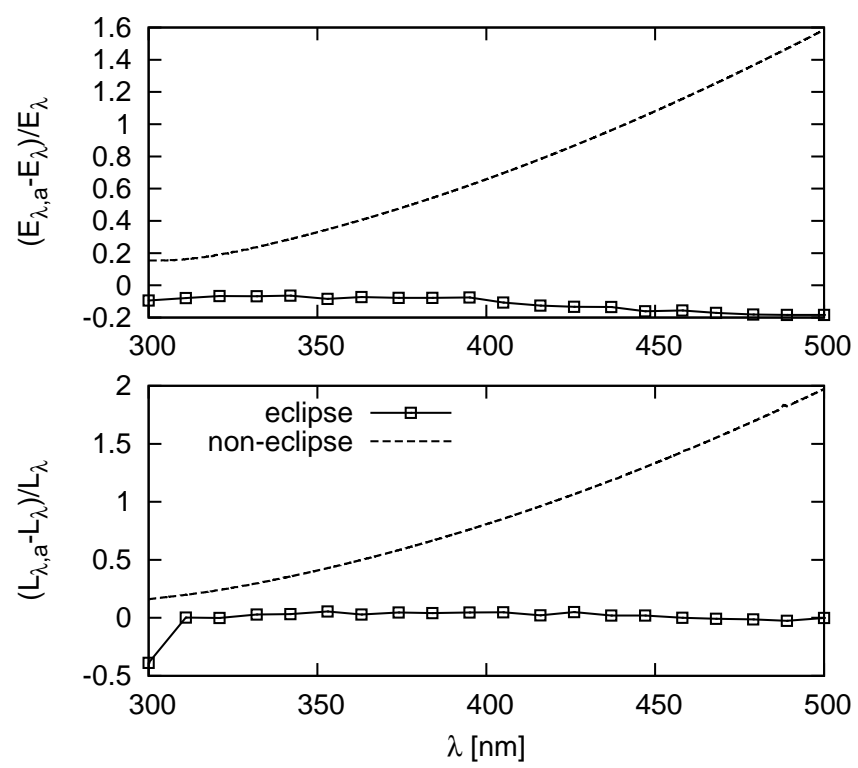

Fig. 12. Impact of aerosol. Relative difference between diffuse irradiance (top) and zenith radiance (bottom) with aerosol $\left(E_{\lambda, a}, L_{\lambda, a}\right)$ and without aerosol. The solid line is for the solar eclipse and the dashed line is for non-eclipse conditions.

however, that volcanic aerosol in the stratosphere could have a large impact on the radiance and irradiance under the umbra.

\subsubsection{Time series}

The radiation at any given time may be simulated from a single backward Monte Carlo calculation if the distribution of photons leaving TOA has been stored. This distribution, weighted by the distribution of incoming solar irradiance for the actual location of the shadow at a given time provides the radiance or irradiance at the sensor for this particular time. Table 1 shows data from Espenak and Anderson (2004) including the exact position of the centre of the umbra every $5 \mathrm{~min}$. Please note, however, that this method may only be applied for short time intervals because solar zenith and azimuth angles $\left(\theta_{0}\right.$ and $\left.\phi_{0}\right)$ change with time, resulting in a different photon distribution at TOA and in a different shape of the shadow. Furthermore the ratio between apparent sun and moon disks $\rho$ varies with time, see Table 1 . This means that for larger time scales the weighting function requires more modifications than just a displacement and the contribution functions needs to be recalculated. Figure 13 shows the time dependence of irradiance and radiance from $400 \mathrm{~s}$ before to $400 \mathrm{~s}$ after totality for three different wavelengths. For this simulation, the parameters $\theta_{0}, \phi_{0}$ and $\rho$ are assumed to be constant, using their value at $0 \mathrm{~s}$. Aerosol is included in this calculation. The horizontal lines are the non-eclipse values for diffuse irradiance and zenith radiance. Irradiance and radiance look similar for 342 and $500 \mathrm{~nm}$ - both wavelengths
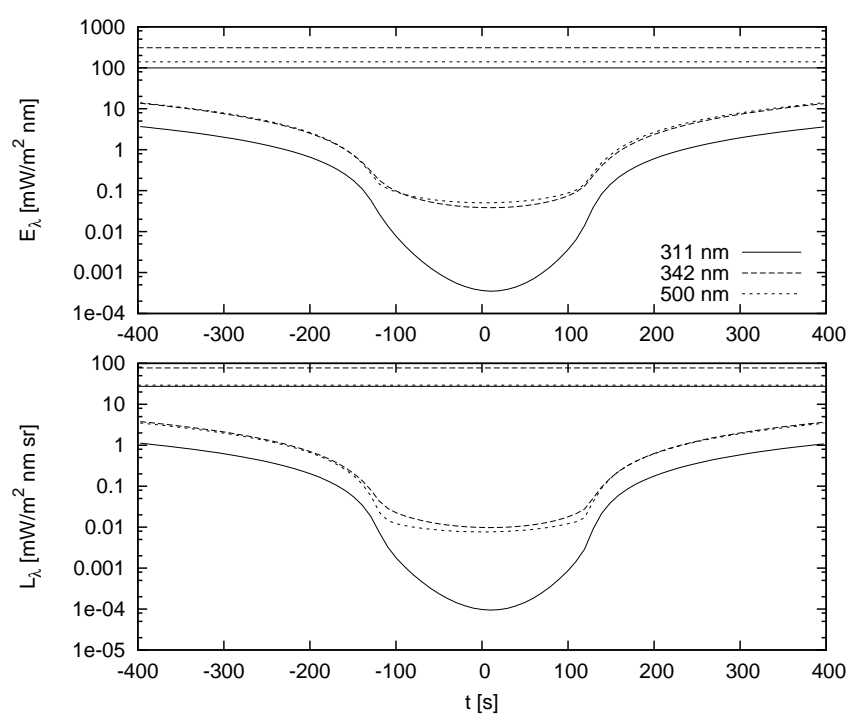

Fig. 13. Simulated time series. $t=0 \mathrm{~s}$ denotes the time when the centres of apparent moon and sun disk coincide.

with only little atmospheric absorption. In the zone of totality $(-80 \mathrm{~s}<t<80 \mathrm{~s})$ there is only a small decrease towards the centre of the shadow, $t=0 \mathrm{~s}$. For $311 \mathrm{~nm}$ radiance and irradiance are much smaller due to the strong ozone absorption and the values decrease strongly towards $t=0 \mathrm{~s}$. This shows that for absorbing wavelengths the distance from the observer to the border of the umbra is very important for the result while for non-absorbing wavelengths light levels are relatively homogeneous under the umbra (please note the logarithmic scale of the plot, however). The curves are nonsymmetric about $\mathrm{t}=0$. This is explained by the photon distribution shown in Fig. 5. The moon shadow travels roughly from from South-West to North-East; this implies that the line between $P 1$ and $P 2$ (from where most of the photons receiving the detector under non-eclipse conditions originated, see Fig. 6) is covered by the elliptical moon shadow after the eclipse but not before the eclipse.

\subsubsection{Three-dimensional radiative transfer effects near the border of the umbra}

Radiation under the umbra can obviously only be calculated with a three-dimensional radiative transfer model which considers horizontal photon transport. Here we investigate how horizontal photon transport affects radiance and irradiance outside but close to the umbra; or in other words, we test the validity of one-dimensional approaches like the one by Koepke et al. (2001). For that purpose we compared our 3-D simulations with a 1-D approximation, scaling the non-eclipse Monte Carlo result with the weighting function Eq. (4) exactly as in Koepke et al. (2001). Both calculations, 1-D and 3-D, were performed assuming a constant sun position which has no impact on the conclusions. 

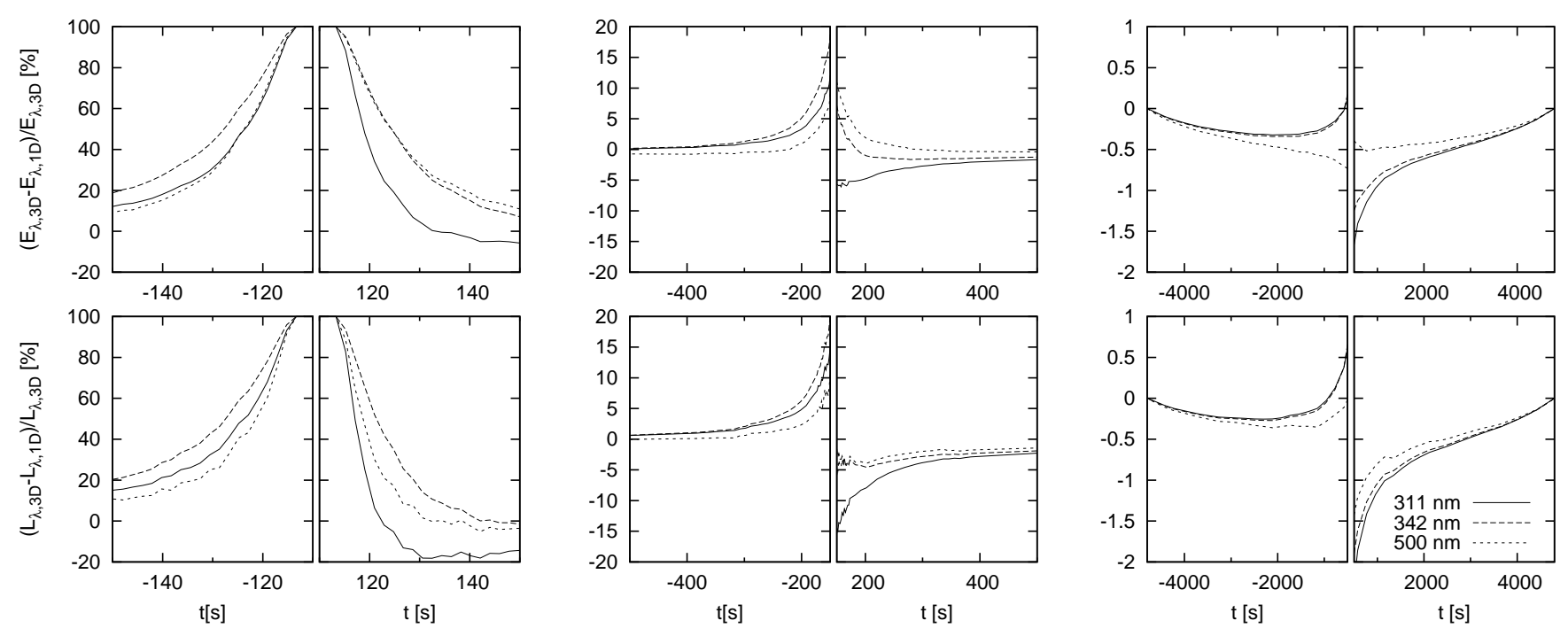

Fig. 14. Comparison between $1-\mathrm{D}$ and 3-D calculations. Relative differences obtained shortly before and after totality. $t=0 \mathrm{~s}$ denotes the time when the centres of apparent moon and sun disk coincide.

The relative differences between the 1-D and the 3-D calculations are shown in Fig. 14 as a function of time, where $t=0$ denotes the time when the centres of moon and sun disc coincide. The upper panels show the irradiance and the lower panels the radiance calculations. $t$ is negative before and positive after the eclipse. The left panels show the relative difference from 110 to $150 \mathrm{~s}$ where $\pm 113.5 \mathrm{~s}$ corresponds to the times of second and third contacts, respectively. The relative difference is $100 \%$ for $-113.5 \mathrm{~s}<t<113.5 \mathrm{~s}$, because the 1-D calculation gives 0 in the umbra. The difference decreases rather quickly, but at $t= \pm 150 \mathrm{~s}$ it is still larger than $10 \%$ for irradiances at $342 \mathrm{~nm}$ and $500 \mathrm{~nm}$. The irradiance is larger in the 3-D calculation, because the weighting function for the extraterrestrial irradiance increases strongly with distance from the umbra; hence there is a significant net horizontal photon transport towards the umbra. The relative difference for zenith radiance decreases faster and for $311 \mathrm{~nm}$ the 3-D calculation becomes clearly smaller compared to the 1-D calculation (almost 20\% at $t=130 \mathrm{~s}$ ). This is due to the asymmetry about $t=0$ (cp. Fig. 13), which has been explained in the previous section. The middle panels show the differences for $\pm(150 \mathrm{~s} \leq t \leq 500 \mathrm{~s})$. Here the difference between 3-D and 1-D decreases from about $15 \%$ to about $1 \%$. In the range $\pm(500 \mathrm{~s} \leq t \leq 4800 \mathrm{~s})$ the difference vanishes slowly. For the case under consideration this implies that about $10 \mathrm{~min}$ "away from totality" the 1-D model can be safely used because the related uncertainty drops below $1 \%$. This might be different for large solar zenith angles, though.

\subsubsection{Influence of the corona}

The corona of the sun is clearly visible in photographs taken during total eclipses. Here we study the contribution of the corona to the radiance and irradiance at the ground. This contribution might be important because the corona is the only source of light reaching the detector directly.

A formula describing the contribution of the corona to the incoming solar irradiance was derived empirically by November and Koutchmy (1996):

$$
\frac{I_{c}(R)}{I_{0}}=10^{-6}\left(\frac{3.670}{R^{18}}+\frac{1.939}{R^{7.8}}+\frac{0.0551}{R^{2.5}}\right)
$$

where $I_{c}$ is the radiance of the corona, $I_{0}$ is the radiance coming from the centre of the solar disk, and $R$ is the distance from the centre. $R$ is normalised to the radius of the sun $R_{S}$, hence $R>1$. To estimate the maximal corona effect this formula has been integrated numerically from $R=1$ to $R=2$, where the corona radiance is already decreased by two orders of magnitude. Since the radiance decreases more than exponentially with distance and the measurements used to derive Eq. (5) were performed only up to $R=1.7$, it is appropriate to integrate up to $R=2$. The result of the integration is $I_{c}^{\text {tot }} \approx 1.7 \cdot 10^{-7} I_{0}$. In order to estimate additional radiation from the corona, this value is added to the weighting function Eq. (4). Since the corona is always visible all photons at TOA get an additional weight corresponding to the contribution of the corona. The relative difference between calculations with and without corona are shown in Fig. 15. For wavelengths larger than $330 \mathrm{~nm}$ the difference is less than $0.1 \%$. Only for short wavelengths close to $300 \mathrm{~nm}$, where the non-corona radiation is almost completely absorbed along the long horizontal path through the atmosphere, the corona has a significant effect. But the radiance or irradiance at this wavelength is still too small to be detected with common instruments anyway. 

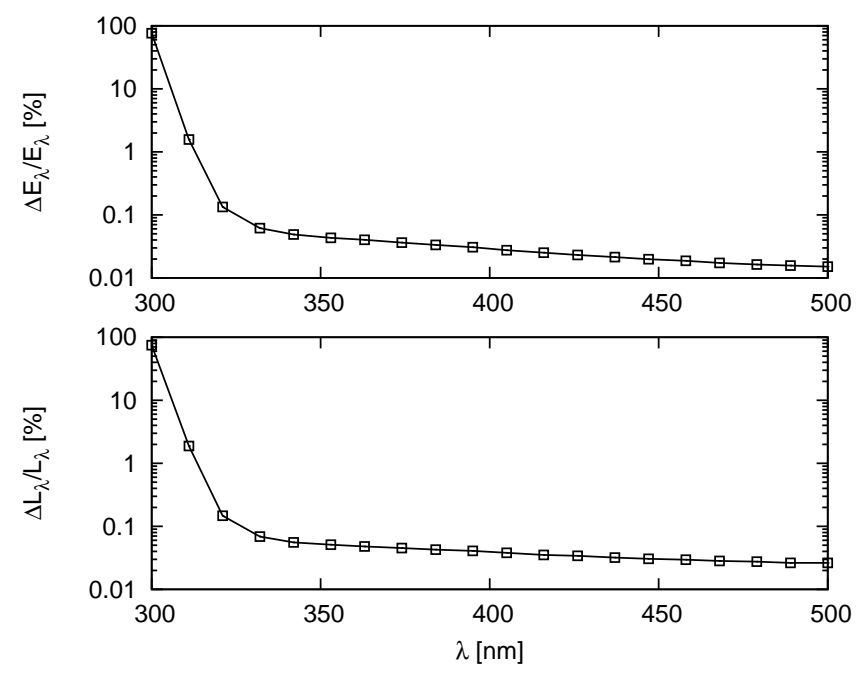

Fig. 15. Additional radiation by corona. Relative difference between simulations with and without corona radiation.

\subsubsection{Colours of the sky}

It is well known from observations that during a solar eclipse the sky looks similar to a sunset all around the horizon. To simulate the sky color we calculated radiance distributions for the complete visible wavelength region 380 to $780 \mathrm{~nm}$ and converted them to RGB values following Walker (2003). A photograph taken by Marthinusen (available at http://www.spaceweather.com/eclipses/29mar06) and the result of the simulation are shown in Fig. 16. The obvious similarity between photograph and simulation indicates nicely that the three-dimensional spherical backward Monte Carlo model developed for this study reproduces the wavelength dependency of the sky radiance successfully.

\section{Conclusions}

Our simulations have shown that the backward Monte Carlo method is well suited for solar eclipse simulations, especially to model irradiances and radiances in the umbral shadow or close to it. The obtained results are of the same order of magnitude as estimated by using a greatly simplified model, which takes into account only first and second order scattering processes (Shaw, 1978). Our results are much more accurate because we take into account multiple scattering. In most previous solar eclipse modelling studies only radiation in the pre-umbra was calculated. We showed that 1-D approximations used in previous studies give accurate results at some distance of the umbra but become more inaccurate close to the border of the umbra before they completely fail below the umbra. The impact of aerosol is smaller in the umbra of an eclipse compared to normal non-eclipse conditions. We could clarify that the radiation emerging from the corona does not affect the radiation

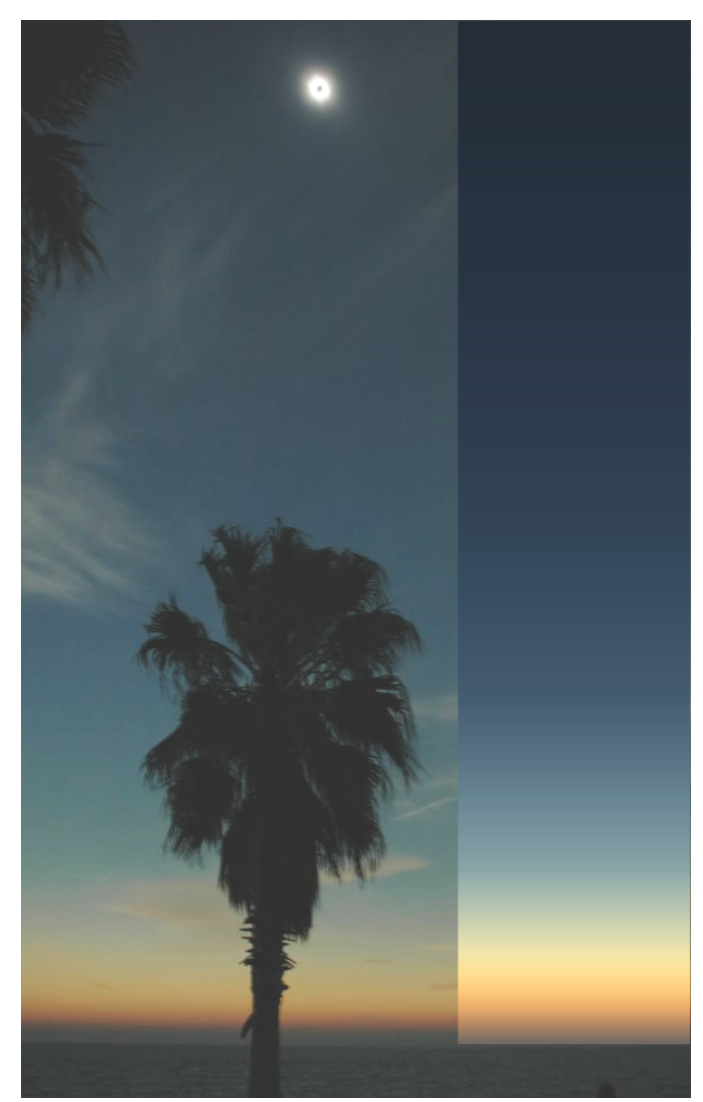

Fig. 16. Reality vs. simulation. The photograph was taken by Marthinusen at 29 March 2006. The simulated Colours of the sky are inserted in the right part of the image.

reaching the umbra significantly. The modelled irradiance and radiance spectra show that radiation measurements in the umbra are very challenging because the total irradiance is decreased by about a factor of 17000 at $340 \mathrm{~nm}$ and even more above $340 \mathrm{~nm}$. The diffuse irradiance or radiance are reduced by a factor of about 5000. Because of the strong ozone absorption in the UV-B, almost no radiation reaches the centre of the umbra in this wavelength region. We hope that these results are helpful for planning future radiation experiments and offer to provide calculations for future eclipses, to help optimising the observations.

Edited by: C. Zerefos

\section{References}

Anderson, G., Clough, S., Kneizys, F., Chetwynd, J., and Shettle, E.: AFGL Atmospheric Constituent Profiles (0-120 km), Tech. Rep. AFGL-TR-86-0110, AFGL (OPI), Hanscom AFB, MA 01736, 1986.

Aplin, K. L. and Harrison, R. G.: Meteorological effects of the eclipse of 11 August 1999 in cloudy and clear conditions, Proc. R. Soc. Lond. A, 459, 353-371, 2003. 
Blumthaler, M., Bais, A., Webb, A., Kazadzis, S., Kift, R., Kouremeti, N., Schallhart, B., and Kazantzidis, A.: Variations of solar radiation at the Earth's surface during the total solar eclipse of 29 March 2006, in: SPIE Proceedings, Stockholm, 2006.

Cahalan, R., Oreopoulos, L., Marshak, A., Evans, K., Davis, A., Pincus, R., Yetzer, K., Mayer, B., Davies, R., Ackerman, T., H.W., B., Clothiaux, E., Ellingson, R., Garay, M., Kassianov, E., Kinne, S., Macke, A., O'Hirok, W., Partain, P., Prigarin, S., Rublev, A., Stephens, G., Szczap, F., Takara, E., Varnai, T., Wen, G., and Zhuraleva, T.: The International Intercomparison of 3D Radiation Codes (I3RC): Bringing together the most advanced radiative transfer tools for cloudy atmospheres, Bull. Am. Meteorol. Soc., 86, 1275-1293, 2005.

Chandrasekhar, S.: Radiative transfer, Oxford Univ. Press, 1950.

Dahlback, A. and Stamnes, K.: A new spherical model for computing the radiation field available for photolysis and heating at twilight, Planet. Space Sci., 39, 671-683, 1991.

Espenak, F. and Anderson, J.: Total solar eclipse of 2006 March 29, Tech. rep., Goddard Space Flight Centre, 2004.

Fabian, P., Winterhalter, M., Rappenglück, B., Reitmayer, H., Stohl, A., Koepke, P., Schlager, H., Berresheim, H., Foken, T., Wichura, B., Häberle, K.-H., Matyssek, R., and Kartschall, T.: The BAYSOFI Campain- Measurements carried out during the total solar eclipse of August 11, 1999, Meteorologische Zeitschrift, 10, 165-170, 2001.

Koepke, P., Reuder, J., and Schween, J.: Spectral variation of the solar radiation during an eclipse, Meteorologische Zeitschrift, 10, 179-186, 2001.

Koutchmy, S.: Coronal physics from eclipse observations, Adv. Space Res., 14, 29-39, 1994.

Marshak, A. and Davis, A. (Eds.): 3D radiative transfer in cloudy atmospheres, Springer, Berlin, Heidelberg, New York, 2005.

Mayer, B.: I3RC phase 1 results from the MYSTIC Monte Carlo model, in: Intercomparison of three-dimensional radiation codes: Abstracts of the first and second international workshops, pp. 49-54, University of Arizona Press, iSBN 0-9709609-0-5, 1999.
Mayer, B.: I3RC phase 2 results from the MYSTIC Monte Carlo model, in: Intercomparison of three-dimensional radiation codes: Abstracts of the first and second international workshops, pp. 107-108, University of Arizona Press, iSBN 0-9709609-0-5, 2000.

Mayer, B. and Kylling, A.: Technical Note: The libRadtran software package for radiative transfer calculations: Description and examples of use, Atmos. Chem. Phys., 5, 1855-1877, 2005, http://www.atmos-chem-phys.net/5/1855/2005/.

Mayer, B., Kylling, A., Madronich, S., and Seckmeyer, G.: Enhanced absorption of UV radiation due to multiple scattering in clouds: experimental evidence and theoretical explanation, J. Geophys. Res., 103, 31 241-31 254, 1998.

November, L. J. and Koutchmy, S.: White-light coronal dark threads and density fine structure, Astrophys. J., 466, 512-528, 1996.

Sharp, W. E., Silverman, S. M., and Lloyd, J. W. F.: Summary of sky brightness measurements during eclipses of the sun, Appl. Opt., 10, 1207-1210, 1971.

Shaw, G. E.: Sky brightness and polarization during the 1973 African eclipse, Appl. Opt., 14, 388-394, 1975.

Shaw, G. E.: Sky radiance during a total solar eclipse: a theoretical model, Appl. Opt., 17, 272-278, 1978.

Shettle, E.: Models of aerosols, clouds and precipitation for atmospheric propagation studies, in: Atmospheric propagation in the uv, visible, ir and mm-region and related system aspects, no. 454 in AGARD Conference Proceedings, 1989.

Silverman, S. M. and Mullen, E. G.: Sky brightness during eclipses: a review, Appl. Opt., 14, 2838-2843, 1975.

Walker, J.: Colour Rendering of Spectra, http://www.fourmilab.ch/ documents/specrend, 2003. 